\title{
Technikfolgen-Abschätzung - eine Herausforderung für die Geographie?
}

Seit einigen Jahren befassen sich Wissenschafterinnen und Wissenschafter mit Technikfolgen-Abschätzungen. Dabei sind hauptsächlich Arbeiten aus der Soziologie, der Philosophie und Politologie anzutreffen (BERGER. 1991; BÖTTGER/FIEGUTH, 1992; LENK/ROPOHL, 1987; METTLER-MEIBOM, 1986; RAPP/MAI, 1989). Sie thematisieren aus unterschiedlichen Blickwinkeln die Folgen einer Technologie für Mensch und Gesellschaft. Der Ethik kommt dabei häufig ein besonderer Stellenwert zu. Sind beispielsweise einzelne gentechnische Anwendungen ethisch vertretbar, und welche Folgen ergeben sich daraus für unsere Gesellschaft?

Die Frage nach den sozialen und gesellschaftlichen Folgen neuer Technologien beschäftigte in den letzten Jahren auch die Entwickler der Technik selbst. In Ingenieurkreisen wird vermehrt der Ruf laut, die Folgen technischer Entwicklungen über reine Funktionalitäts- oder Wirtschaftlichkeitsüberlegungen hinausgehend zu bedenken. So befaßt sich in der Bundesrepublik der Verein Deutscher Ingenieure (VDI) bereits seit den 80er Jahren mit Technikfolgen-Abschätzung. Er erachtet Technikfolgen-Abschätzung als geeignetes Instrument, um unerwünschte Nebenwirkungen einer Technologie bereits frühzeitig zu erfassen und zu analysieren. Damit werde Technikfolgen-Abschätzung zunehmend "zum Instrument gestalterischer Begleitung technischer Entwicklungen» (VDI-Technologiezentrum 1992, Vorwort).

Neue Technologien werfen nicht nur gesellschaftliche oder technisch-gestalterische Fragen auf. Wirkungen einer Technik ${ }^{1}$ manifestieren sich in den verschiedensten Bereichen wie Wirtschaft, Kultur, Umwelt, Politik und Raum. Am Beispiel der Einführung des Automobils lassen sich die weitreichenden Folgen einer Technikanwendung sehr gut nachvollziehen. Das Automobil hat massive Änderungen im wirtschaftlichen und gesellschaftlichen Bereich gebracht und wesentlich zur Verschlechterung der Umweltsituation beigetragen. Die Auswirkungen dieser Technik auf den Raum sind ebenfalls beträchtlich: Suburbanisierung, Zersiedelung und Kulturlandverlust sind nur einige Stichworte. Damit ist auch gezeigt, daß die Wirkungen einer Technologie nicht nur für Technikspezialisten, Soziologinnen oder Politologen von Interesse sind, sondern auch für Geographinnen und Geographen. Sie sind ebenso angesprochen, wenn es darum geht, Technikfolgen für Gesellschaft, Umwelt und insbesondere für den Raum abzuschätzen. Deshalb sollte die Geographie um eine aktive Rolle im Forschungsfeld der Technikfolgen-Abschätzung bemüht sein.
Im folgenden soll die Entstehungsgeschichte der Technikfolgen-Abschätzung kurz skizziert und sollen ihre Ziele, Inhalte und Funktionen vorgestellt werden. Daran knüpfen Überlegungen zu möglichen Bewertungskriterien an, die mit einer exemplarischen Darstellung aus dem Bereich der Telematik ${ }^{2}$ abgeschlossen werden.

\section{Entwicklung der Technikfolgen-Abschätzung}

Die gesamttechnische Entwicklung wird heute nicht mehr von so großem Vertrauen begleitet wie noch in den $50 \mathrm{er}$ und 60er Jahren. Damals herrschte der Glaube vor, daß beinahe alles technisch machbar sei. Technische Entwicklungen versprachen eine Verbesserung der materiellen Lebensbedingungen und durch eine vernunftgemäße Gestaltung der Rahmenbedingungen auch einen sozialen Fortschritt der Menschheit. Dabei hat es nie an Stimmen gefehlt, die vor Gefahren der Technik gesundheitlicher und moralischer Art gewarnt haben. Meist war es die neueste Technologie, deren Gefährlichkeit diskutiert wurde. Ausschlaggebend war aber ein überwiegendes Vertrauen der Gesellschaft in den Fortschritt der Technik. Dies gründete auch in der Bereitschaft des Einzelnen, den Instanzen zu vertrauen, die diese Technik entwickelten und einführten. Dieses Vertrauen in die Instanzen ist seit den 60er Jahren massiv geschwunden. Heute besteht eher eine Ablehnung oder Angst vor großen Organisationen und Systemen, ein Mißtrauen gegen Staat, Wirtschaft und ihre Repräsentanten.

Dieser Wandel im Verhältnis von Mensch zu Technik läßt sich nach KUHLMANN (1977, 159 f.) mit folgenden Argumenten begründen:

In der heutigen technischen Zivilisation gibt es die einen, die Wesen und Arbeitsweise der Technik verstehen, und die anderen, die nur den Schalter bedienen. Moderne Großtechniken wie die Kernenergietechnik oder die Informationstechnologie sind nur noch für wenige Fachleute durchschaubar. Das hat zu einer scharfen Trennung zwischen den wenigen Wissenden und den vielen Unwissenden geführt. Letztere empfinden sich leicht als einge-

Simone Rangosch-du Moulin, dipl. Geographin, Geographisches Institut, Universität Zürich Irchel, Winterthurerstraße 190, 8057 Zürich 
spannt in ein System, an dessen Zwecken sie nicht beteiligt sind. Daraus folgen häufig Mißtrauen gegenüber technischen Systemen und Kritik an der Technik.

Ein zweites wichtiges Argument KUHLMANNS ist die scheinbare Eigendynamik, die dem Prozeß des technischen Fortschritts innewohnt. Bedeutende Erfindungen und technische Neuerungen folgten in immer kürzeren Zeitabständen. Mit den Diskussionen um die Kernenergie übten in den 70er Jahren erstmals breitere Kreise der Öffentlichkeit Technikkritik, später kam der Problemkreis "Technik und Umweltbelastung" hinzu. Daran schlossen sich in den 80er Jahren die Komplexe «Mikroelektronik und Rationalisierung», "Datenbanktechnik und Persönlichkeitsschutz» und "moderne Telekommunikation und Ausweitung der elektronischen Massenmedien» an.

Schließlich ist seit den 70er Jahren eine immer ausgedehntere Anwendung moderner Techniken und ein Trend zu größeren Anlagen zu beobachten, was die schädlichen Nebenwirkungen, Gefahrenpotentiale und Umweltbelastungen der Technik zum Teil tatsächlich verstärkt hat, zum Teil aber auch nur im Bewußtsein der Öffentlichkeit stärker hervortreten ließ. Damit wuchs die Angst vor Katastrophen, die gerade bei der Kerntechnologie durch zahlreiche Zwischenfälle und den folgenschweren Reaktorunfall in Tschernobyl 1986 verstärkt wurde. Darauf wurden in den 80er Jahren die Forderungen nach dem Schutz von Mensch und Umwelt vor den Gefahren der Technik immer lauter.

Vor dem Hintergrund der Kernenergiedebatte und der wachsenden Technikkritik entstand in den USA die Idee, negative Folgen von Technologien möglichst vor ihrer Einführung abzuklären, d. h. mit wissenschaftlichen Instrumenten mögliche Effekte des Technikeinsatzes abzuschätzen. 1966 veröffentlichte das «Subcommittee on Science, Research and Development» des US-Repräsentantenhauses einen Bericht über die Nebenwirkungen technischer Innovationen. Dieser Bericht forderte unter anderem die Einrichtung eines "Early Warning System» zur Entdeckung negativer und positiver Folgewirkungen von Technikanwendungen (PETERMANN, 1991, 19). In diesem Bericht fand der Begriff "Technology Assessment» wohl erstmals offiziell Verwendung. In der Folge wurden in den USA eine Reihe exemplarischer Technology-Assessment-Fallstudien durchgeführt und schließlich 1972 das "Office of Technology Assessment» (OTA) des USKongresses gegründet. Diese Institution ist bis heute von ihrer Größe und von der institutionellen Konstruktion her einzigartig geblieben. Das OTA sollte die Auswirkungen von neuen Technologien bei technischen Großprojekten im voraus abschätzen und damit der Legislative, dem US-Kongreß, Entscheidungshilfen bei der Einführung und Anschaffung neuer Technologien bieten. Unter den möglichen Folgen sollten vor allem jene Effekte abgeschätzt werden, die über rein wirtschaftliche Rentabilitätsüberlegungen hinausgehen, $d . h$. welche insbesondere die Lebens- und Umweltqualität beeinflussen könnten.
Technology Assessments wurden bald darauf auch in anderen Industrieländern bekannt und beeinflußten dort die forschungs- und technologiepolitischen Diskussionen. In der Bundesrepublik Deutschland wurde die Idee des Technology Assessment zu Beginn der 80er Jahre von BÖHRET Und FRANZ, PASCHEN, GRESSER, CONRAD und anderen aufgegriffen und unter dem Namen "Technologiefolgen-Abschätzung» oder "Technikfolgen-Abschätzung» (TA) bekannt. In der Literatur sind auch Bezeichnungen wie Technikbewertung oder Technikfolgenbewertung, Technikfolgenforschung, Technikwirkungsforschung usw. anzutreffen, wobei Technik wahlweise durch Technologie ersetzt wird. Die Vielfalt der Begriffe entstand aufgrund der Schwierigkeit der Übersetzung des englischen Begriffs Technology Assessment. Er beinhaltet einerseits die vorausschauende Abschätzung und andererseits die Bewertung einer Technologie.

PASCHEN/PETERMANN (Zitat in: PETERMANN, 1991, 20) umschreiben die Ziele einer Technikfolgen-Abschätzung wie folgt:

- «die Bedingungen und (potentiellen) Auswirkungen der Einführung und (verbreiteten) Anwendung von Techniken systematisch zu erforschen und zu bewerten,

- gesellschaftliche Konfliktfelder, die durch den Technikeinsatz entstehen können, zu identifizieren und zu analysieren und

- Handlungsmöglichkeiten zur Verbesserung der betrachteten Technik bzw. ihrer Anwendungsmodalitäten aufzuzeigen und zu überprüfen (policy analysis)».

Ihrem Ursprung und Konzept nach ist TechnikfolgenAbschätzung ein Analyse- und Bewertungsansatz bezüglich der Voraussetzungen und Folgenpotentiale des gesellschaftlichen Einsatzes von Techniken. Obwohl in der deutschsprachigen Literatur oft als solches bezeichnet, ist Technology Assessment kein Verfahren, denn es beschreibt keine verbindliche, routinemäßige Vorgehensweise. Es ist vielmehr ein strategisches Rahmenkonzept. Dieses Konzept wird in den verschiedenen Ländern unterschiedlich ausgelegt. Die Technikfolgen-Abschätzungen variieren im Hinblick auf Funktion und Inhalt.

Eine mögliche Funktion - wie sie beispielsweise vom amerikanischen Office of Technology Assessment wahrgenommen wird - ist die Erweiterung der Grundlagen für eine Technologiepolitik, indem die TechnikfolgenAbschätzung den Entscheidungsträgern breitere Informationen über mögliche Folgen geben soll. So wurden beispielsweise soziale Folgen von Telearbeit wie Isolation oder arbeitsrechtliche Benachteiligung untersucht und in die politische Diskussion eingebracht (BALLER. STEDT, 1982; SCHWOHNKE/WICKE, 1986; LENK. 1989). Ziel kann dabei eine Vergrößerung des Personenkreises innerhalb des Entscheidungsprozesses sein oder gar der Einbezug einer breiteren Öffentlichkeit. Das heißt, die Abschätzung von Technikfolgen kann durch umfassende sachliche Information über den Kreis der Fachleute hinaus zu einer breiten Diskussion und einer allgemeinen Bewußtseinsbildung in bezug auf den Einsatz von Tech- 
nologien beitragen. Mit der Information der Öffentlichkeit kann auch die Absicht verbunden sein, die gesellschaftliche Akzeptanz neuer Technologien - beispielsweise neuer Kommunikationstechniken - zu erhöhen. Andere Technikfolgen-Abschätzungen haben die Funktion, Aussagen zu einer sozialen Technikgestaltung zu liefern, d. h. sozial wünschenswerte Technikanwendungen zu formulieren und zu fördern. Diese Funktion der Technikfolgen-Abschätzung ist besonders in den Niederlanden anzutreffen (BUCHS, 1992, 15). Mit Fragen der sozialen Gestaltung einer Technik haben sich im Falle der Telematik unter anderen wITT-BARTHEL. 1992, und der Deutsche Gewerkschaftsbund (DGB-Bundesvorstand, 1991) befaßt.

Wieder andere Technikfolgen-Abschätzungen halten an der ursprünglichen Idee des Technology Assessment fest, mit dem Ziel, ein Frühwarnsystem aufzubauen. Dieses «Early Warning System» soll Probleme einer Technologie vorihrer Einführung abschätzen und vor negativen Wirkungen warnen.

\section{Kontroverse Diskussionen um Technikfolgen-Abschätzungen}

Auf den ersten Blick scheint Technikfolgen-Abschätzung eine allgemein einleuchtende, attraktive Aufgabe zu sein, und ihre Durchführung verspricht beträchtlichen gesamtgesellschaftlichen Nutzen. Die zunehmende Bedrohung vieler Bereiche der Gesellschaft und der natürlichen Umwelt durch unvorhergesehene Neben- oder Spätwirkungen von Techniken verdeutlicht dies. So wurde beispielsweise bei der Einrichtung von elektronischen Bankschaltern und -automaten in vielen Fällen nicht beachtet, daß das Bedienfeld für Menschen im Rollstuhl auBer Reichweite ist, womit diese Menschen im Falle eines totalen Ersatzes des Schalterpersonals durch Automaten enorme Nachteile zu tragen hätten. Automation und Einführung von Computern werfen auch gravierende ökologische Probleme auf: Die Zusammensetzung heutiger Telematikendgeräte, die aus einem Gemisch verschiedenster Stoffe bestehen - Kunststoffe, Metalle, Glas usw. -, erschwert ein Recycling. Durch die schnelle Veraltung der Computer, aber auch der Faxgeräte und Drucker wird das Phänomen "Computerschrott» zum künftigen Entsorgungsproblem. Zudem werden bei der Herstellung von Mikrochips, einer wesentlichen Basis vieler Telematikeinrichtungen, unter anderem Gifte wie Arsin, Phosphin und Fluorchlorkohlenwasserstoffe (FCKW) verwendet (HENCKEL, 1990, 55 f.).

Die wachsende Komplexität und Größenordnung neuer Techniken mit immer schwerer durchschaubaren Auswirkungsketten rufen direkt nach einer Ex-ante-Abschätzung der Technikfolgen. Daß dennoch die öffentliche Diskussion über Technikfolgen-Abschätzungen von Anfang an kontrovers verlaufen ist, läßt sich aus der Unterschiedlichkeit der Interessenlagen verschiedener gesellschaftlicher Gruppen erklären. Von seiten der Industrie, aber auch von staatlichen Stellen wurde und wird häufig befürchtet, eine breite Anwendung des TA-Konzeptes würde den technischen Fortschritt und damit auch das wirtschaftliche Wachstum hemmen. Daß also aus dem "Technology Assessment» letztlich ein "Technology Arrestment" wird, das Innovatoren abschrecken, technische Entwicklungen und Anwendungen behindern und blokkieren würde. Hinzu kommt die Befürchtung, daß durch das Aufzeigen von möglichen Folgen ein Klima der Angst geschaffen wird, welches dann Teile der Bevölkerung zur Akzeptanzverweigerung bei technischen Neuerungen veranlaßt. Das Ziel der Technikfolgen-Abschätzung ist aber nicht die Behinderung, sondern eine reflektierte Gestaltung soziotechnischer Systeme und das Aufzeigen potentieller Gefahren des Einsatzes von Techniken. Solche Gefahren bleiben bei herkömmlichen Planungs- und Bewertungsverfahren - wie z. B. Investitionsrechnungen, Markt- oder Kosten-Nutzen-Analysen - in der Regel unbeachtet.

\section{Technology Assessment in der Schweiz}

Die Art der Diskussion erinnert an die Debatte über die Umweltverträglichkeitsprüfungen (UVP) in der Schweiz. Auch die UVP, die sich mit den möglichen Folgen eines geplanten Projektes befaßt und sie unter dem Gesichtspunkt der Umweltverträglichkeit bewertet, wurde und wird heute wieder verstärkt als Verhinderungsinstrument, als staatlicher Eingriff in die unternehmerische Freiheit und als Feind der Wettbewerbsfähigkeit kritisiert. Eine öffentliche Debatte über Technikfolgen-Abschätzungen blieb in der Schweiz allerdings bisher aus, und auch die Diskussionen im nahen Ausland berührten nur wenige Interessierte aus Politik und Wissenschaft. Öffentlich thematisiert wurden sie erst mit der Botschaft des Bundesrates vom 9. Januar 1991 betreffend die «Förderung der wissenschaftlichen Forschung in der Periode von 1992 bis 1995" und einem "Aktionsprogramm auf dem Gebiet der Mikroelektronik», als der Bundesrat dem Schweizerischen Wissenschaftsrat den Auftrag erteilte, "... bis zum Ende der Legislaturperiode 1992-95 ein Instrumentarium der Technikfolgenabschätzung (TA) vorzubereiten und während dieser Versuchsperiode bereits erste Studien durchzuführen" (Zitat: SWR 1992, 2). Daraufhin schrieben die Verantwortlichen für Wissenschaft und Forschung Ende 1992 das sogenannte Forschungsprogramm "Technology Assessment - Programm TA» aus. In dieser Ausschreibung bezeichnet der Schweizerische Wissenschaftsrat die Funktion der TechnikfolgenAbschätzung mit einer Definition von VARY COATES vom amerikanischen Office of Technology Assessment: «... It is the systematic identification, analysis and evaluation of the potential consequences (whether benefical or detrimental) of technology in terms of its impact on social, cultural, political, economic and environmental systems and processes. Its goal is to inform the policy process by putting before the decisionmaker an analyzed set of options, alternatives and consequences ... it is not the deci- 
sion process itself, but only one input into that process.» (Zitat: COATES, in: SWR 1992, 3).

Zu den ersten Prioritäten des Programms TA gehörte es, Musterstudien zu unterschiedlichen konkreten Themen in Anlehnung an die laufenden Schwerpunktprogramme zu erstellen sowie einen Dokumentationsdienst und ein Inventar über TA-Aktivitäten und -Fachleute aufzubauen. Innerhalb des vergleichsweise engen finanziellen Rahmens des Programms, der für die Jahre 1992 bis 1995 rund 3,4 Millionen Franken betrug, waren die Jahre 1993 und 1994 als Versuchsphase angelegt, in welcher mehrere Technikfolgen-Abschätzungen für verschiedene Technologien geplant waren. Diese Phase sollte Aufschlußgeben über Konzepte, Bewertungsverfahren und anzuwendende Methoden. Im laufenden Jahr wird dann entschieden werden, wie und in welchem Rahmen TechnikfolgenAbschätzungen in der Schweiz durchgeführt und institutionalisiert werden können.

$\mathrm{Zu}$ Beginn des Programms TA waren die konkretesten Vorstellungen zu Technikfolgen-Abschätzungen im Schwerpunktprogramm Biotechnologie anzutreffen. Unter dem Aspekt der biologischen Sicherheits- und Risikoforschung, die sich insbesondere mit Gesundheit und Umweltschutz befaßt, waren verschiedene Studien in Vorbereitung. In eine TA-Untersuchung sollten jedoch auch Überlegungen zu den gesellschaftlichen Auswirkungen der Technologie einbezogen werden. Während die Biotechnologie einerseits durch gentechnisch verändertes Saatgut eine Reduktion des Pestizideinsatzes bewirken und damit die Umwelt entlasten kann, sind andererseits die sozialen Folgen - zum Beispiel für Landwirte - nicht nur positiv. Pflanzen, die aus gentechnisch verändertem Saatgut gezogen wurden, können ihre Resistenz gegen Schädlinge nicht an die nächste Generation weitergeben. Damit sind die Landwirte abhängig von den Produzenten des Saatguts, das immer neu im Labor hergestellt und für jede Aussaat eingekauft werden muß. Diese neue Abhängigkeit und weitere soziale Folgen von Biotechnologie-Anwendungen müssen durch eine Technikfolgen-Abschätzung benannt werden.

\section{Technikfolgen-Abschätzung als Umwelt- und Sozialverträglichkeitsprüfung}

Technology Assessment geht also einen Schritt weiter als die biologische Risikoforschung oder die Umweltverträglichkeitsprüfungen, indem sie nicht nur die Sicherheit und Umweltverträglichkeit einer Technologie und ihrer Anwendungen prüft, sondern ebenso die Folgen für das Individuum und die Gesellschaft abschätzen und bewerten soll. Es geht im Kern also um eine Umwelt- und Sozialverträglichkeitsprïfung. Das heißt, positive und negative Auswirkungen auf ökologische, soziale, politische und wirtschaftliche Systeme und Abläufe sollen abgeschätzt werden. Weil die Wirkungen einer Technologie in möglichst allen Bereichen abgeschätzt werden sollen dazu gehören die Bereiche Gesellschaft, Umwelt, Kultur,
Politik, Wirtschaft, Recht, Verwaltung und Raum -, ist eine interdisziplinäre Zusammenarbeit nötig. Um Technikfolgen-Abschätzungen umfassend zu realisieren, ist auch die Geographie gefordert, ihre Erfahrungen aus räumlichen, sozioökonomischen und ökologischen Analyse- und Bewertungsverfahren einzubringen. Mit ihrem Spezialwissen und der Fähigkeit zur Synthese von Erkenntnissen verschiedener Fachbereiche können Geographinnen und Geographen wertvolle Mitarbeit leisten in einem interdisziplinären Team zur Technikfolgen-Abschätzung.

Die Umweltverträglichkeitsprüfungen waren ein erstes Aufgabengebiet, das es nun zu erweitern gilt. Während der Ausdruck «Umweltverträglichkeit» durch die UVP in den letzten Jahren Bekanntheit erlangte, ist der Begriff «Sozialverträglichkeit» weniger bekannt. Es ist zu klären, was hinter diesem Begriff steht und welche Bedeutung er im Zusammenhang mit der Einführung neuer Technologien hat. Vereinfacht läßt sich sagen, daß eine Technologie und deren Anwendungen dann sozialverträglich sind, wenn sie für Mensch und Gesellschaft nicht schädigend sind. Zur Beurteilung der Sozialverträglichkeit muß bereits der Zugangzu einer Technologie herangezogen werden (DU MOULIN/KLAUS. 1993, 53 ff.). Ist dieser Zugang für alle Menschen gleichermaßen möglich, oder werden einzelne soziale Gruppen, z. B. durch hohe Kosten oder hohe Anforderungen an manuelle oder geistige Fähigkeiten, von der Benützung einer Technik ausgeschlossen? Im Falle der Telematik betrifft dies die Übermittlungs- und Benutzungsgebühren der Kommunikationsmedien und die Komplexität der Kommunikationsvorgänge. Es stellt sich zudem die Frage, ob die Anwendung einer Technologie zu Diskriminierungen bestimmter Gruppen, z. B. behinderter, alter oder ärmerer Menschen, führt, indem künftig für persönlich erbrachte Dienstleistungen höhere Gebühren zu entrichten sind als für medial erbrachte (z. B. Telebanking).

Solche Fragen müssen bei der Untersuchung der Sozialverträglichkeit abgeklärt werden. Dazu gehören auch die Themen Mitbestimmung und Unabhängigkeit. Mitbestimmung beispielsweise bei der Einführung von Computern am Arbeitsplatz und bei Entscheidungen für oder gegen Systeme zur Prozeßüberwachung und Leistungskontrolle. Die Abhängigkeit von Technologien oder einzelnen Anwendungen muß ebenfalls hinterfragt werden. Ist z. B. das Funktionieren des Gesundheitswesens in Zukunft abhängig vom reibungslosen Ablauf der Datenübertragung zwischen Ärzten und Spitälern oder vom fehlerlosen Speichern der Patientendaten?

\section{Bewertungsproblematik und Bewertungskriterien}

Wenn eine Technikfolgen-Abschätzung die Sozial- und Umweltverträglichkeit von Technologien und deren Anwendungen beurteilen soll, hat sie auch zu benennen, was überhaupt sozial respektive ökologisch verträglich ist und was nicht. Das verlangt in einem ersten Schritt eine Abschätzung möglicher Technikfolgen und in einem 
zweiten Schritt deren Bewertung. Die Frage nach den Kriterien einer Technikbewertung war und ist umstritten, denn die Meinungen darüber, was sozial- und umweltverträglich ist, gehen weit auseinander. Am ehesten können noch Gemeinsamkeiten in bezug auf die Umweltverträglichkeit gefunden werden. Die Beurteilung sozialer Auswirkungen einer Technologie ist demgegenüber sehr viel anspruchsvoller. Einen Konsens bezüglich der Sozialverträglichkeit zu finden scheint beinahe unmöglich, was in jeder politischen Diskussion offenkundig wird. Für die einen ist eine Erhöhung des individuellen Wohlstandes wichtigstes Ziel, für die anderen sind Gesundheit und soziale Sicherheit von größter Bedeutung. Wieder andere möchten einen möglichst großen individuellen Handlungs- und Entscheidungsspielraum erreichen.

Die Verschiedenartigkeit der Technologien, die Vielfalt möglicher Wirkungen und das breite Spektrum gesellschaftlicher und politischer Rahmenbedingungen in den verschiedenen Ländern haben bis heute die Entwicklung eines einheitlichen Konzeptes oder eines verbindlichen Verfahrens für eine Technikbewertung nicht zugelassen. Es kann auch keine allgemeingültigen Bewertungsverfahren geben, genausowenig wie Objektivität im naturwissenschaftlichen Sinne. Eine Bewertung von Technologien ist immer abhängig vom Gesellschafts- und Menschenbild der Bewertenden. Gerade weil die Bewertung subjektiv ist, ist es erforderlich, daß die Werte für eine Technikfolgen-Abschätzung von Beginn einer Untersuchung an offengelegt und transparent gemacht werden. PASCHEN/GRESSER/CONRAD $(1978,15)$ forderten, daß alle Schritte einer Technikfolgen-Abschätzung intersubjektiv nachvollziehbar sein müßten. Sowohl die Auswahl der Bewertungskriterien als auch die Festlegung des Zielsystems sollen offenliegen und einsehbar sein. Annahmen, Auswahlentscheidungen und Werturteile, die zum Ergebnis einer Technikfolgen-Abschätzung führen, müssen von Dritten nachprüfbar sein. Damit soll transparent werden, welche Zielvorstellungen einer TA-Untersuchung zugrunde liegen, weil sich die Bewertungskriterien an diesen Zielvorstellungen über eine wünschenswerte Entwicklung orientieren. Denn erst wenn klar benannt wird, in welche Richtung sich Gesellschaft und Umwelt entwickeln sollen, können die Auswirkungen einer Technologie in ihrem Beitrag zur gewünschten Entwicklung bewertet werden.

Es gibt verschiedene Versuche, die Bewertungskriterien einer Technikfolgen-Abschätzung zu konkretisieren. Der Verein Deutscher Ingenieure schreibt beispielsweise 1986 in seinem Vorentwurf für eine Richtlinie «Empfehlungen zur Technikbewertung», daß die Technik einer Bewertung hinsichtlich der Werte Wohlstand, Wirtschafilichkeit, Funktionsfähigkeit, Sicherheit, Gesundheit sowie Umweltqualität, Persönlichkeitsentfaltung und Gesellschaftsqualität unterzogen werden solle. Der Inhalt dieser Werte wird näher definiert, so z. B. im Bereich Persönlichkeitsentfaltung und Gesellschaftsqualität: Hierzu gehören soziale Sicherheit, Solidarität, Handlungsfreiheit und Gerechtigkeit ebenso wie Beherrschbarkeit der Technik und
Überschaubarkeit technischer Prozesse (Verein Deutscher Ingenieure, 1987, 308-325).

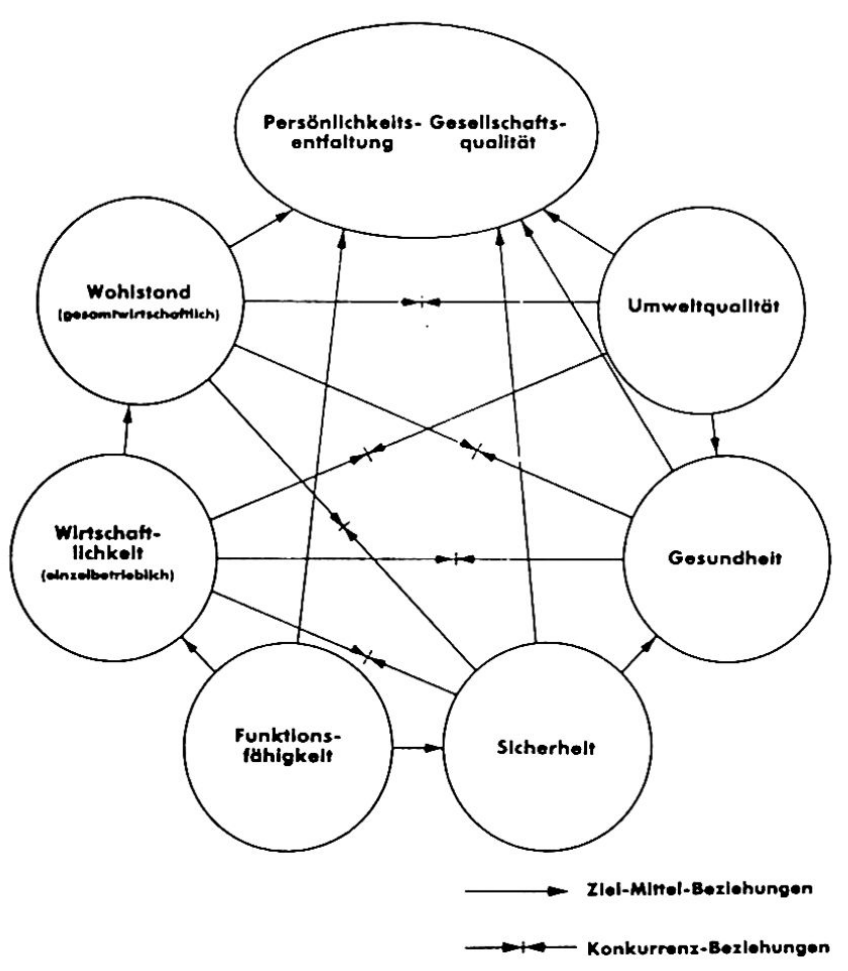

Abb.1 Werte im technischen Handel

(Quelle: Verein Deutscher Ingenieure 1987, 323)

Viele der obigen Bewertungskriterien, zwischen denen offensichtlich auch Zielkonflikte bestehen, sind in gleicher oder ähnlicher Form im Konzept des qualitativen Wachstums enthalten, das ROTACH et al. 1993 in einer TAUntersuchung über Informations- und Kommunikationstechnologien verwendeten. Sie bezogen sich auf das Konzept des qualitativen Wachstums, wie es vom Schweizer Bundesrat zu Beginn der Legislaturperiode 1987-91 als Leitidee seiner Tätigkeit zugrunde gelegt und auch in der Planung der folgenden Legislaturperiode 1991-95 wiederaufgenommen wurde (Schweizerische Bundeskanzlei 1988 u. 1992). Damit konnten sich ROTACH et al. auf politisch konsensualisierte Zielsetzungen hinsichtlich einer gewünschten Entwicklung in der Schweiz abstützen.

Das Konzept des qualitativen Wachstums wurde von der Expertenkommission "Qualitatives Wachstum» im Schlußbericht von 1985 folgendermaßen definiert: "Qualitatives Wachstum ist jede nachhaltige Zunahme der gesamtgesellschaftlichen und pro Kopf der Bevölkerung erreichten Lebensqualität, die mit geringerem oder zumindest nicht ansteigendem Einsatz an nicht vermehrbaren oder nicht regenerierbaren Ressourcen sowie mit abnehmenden oder zumindest nicht zunehmenden Umweltbelastungen erzielt wird. (...) Gesamtwirtschaftlich gesehen bedeutet qualitatives Wachstum eine Orientierung auf einen international wettbewerbsfähigen Typus der Wertschöpfung, der mit weniger externen Kosten und 
Belastungen Dritter sowie geringerem Aufwand an Rohstoffen und Energie verbunden und durch den Einsatz von mehr Fähigkeitskapital gekennzeichnet ist» (Qualitatives Wachstum (BfK), 1985, zit. n. ROTACH et al., 1993, 17). Ausgehend von dieser Definition, die die zwei Komponenten Lebensqualität und Umweltqualität beinhaltet, erarbeiteten ROTACH et al. ein Bewertungskonzept, das nachfolgend in verkürzter Form vorgestellt werden soll.

Lebensqualität, verstanden als die Befriedigung sowohl materieller als auch immaterieller Bedürfnisse, geht über den bloßen wirtschaftlichen Wohlstand hinaus und umfaßt auch das subjektive Wohlbefinden. Eine Steigerung der Lebensqualität im Sinne eines qualitativen Wachstums beinhaltet folgende Hauptziele:

- eine Vergrößerung der Unabhängigkeit als Erweiterung des Entscheidungs- und Handlungsspielraumes für den Einzelnen, für Unternehmen und Regionen,

- die Chancengleichheit, d.h. den Zugang zu Lebenschancen bei Arbeit, Bildung, medizinischer Versorgung usw., sowie den Abbau regionaler Disparitäten,

- eine Erhöhung von Partizipationsmöglichkeiten im Sinne von vermehrter Mitsprache im Betrieb, beim Wohnen, in politischen Körperschaften usw.,

- die Verbesserung der physischen, psychischen und sozialen Gesundheit

- und die Erhöhung der materiellen Sicherheit, z. B. Arbeitsplatz- und Einkommenssicherheit, Kündigungsschutz, Wettbewerbsfähigkeit der Betriebe und regionaler Ausgleich.

Umweltqualität beinhaltet den Umgang mit natürlichen Ressourcen und das Ausmaß der Umweltbelastung. Die Ziele zur Steigerung der Umweltqualität lauten deshalb:

- Reduktion des Ressourcenverbrauchs durch geringeren Rohstoff- und Energieeinsatz,

- Reduktion der Umweltbelastung, indem weniger Abfälle und Emissionen verursacht werden

- und Erhaltung des Lebensraumes für Menschen, Tiere und Pflanzen durch Landschaftsschutz, Kulturlanderhaltung und eine haushälterische Nutzung des Bodens.

Die acht obengenannten Hauptziele müssen weiter in Teilziele differenziert und die Zielkonflikte zwischen den Teilzielen benannt werden. So steht beispielsweise der Arbeitsplatzsicherheit für das Individuum oft die Erhaltung der Wettbewerbsfähigkeit des Betriebes entgegen, oder den Schutzinteressen für die Umwelt werden Flächenansprüche der Betriebe und Haushalte entgegengehalten. Erst nach der Bezeichnung von Teilzielen und der Diskussion der Zielkonflikte können die Bewertungskriterien für eine Technikfolgen-Abschätzung festgelegt werden. Anschließend lassen sich die Wirkungen einer Technik im Hinblick auf die einzelnen Teilziele beurteilen und Aussagen zur Sozial- und Umweltverträglichkeit einer Technologie treffen.

Vor dem Hintergrund des obigen Bewertungskonzeptes sollen am Beispiel der Informations- und Kommunika- tionstechnologien (Telematik) einige Aussagen betreffend Sozial- und Umweltverträglichkeit angeführt werden.

\section{Sozial- und Umweltverträglichkeit der Telematik}

Der Einsatz der Telematik erlaubt auf der einen Seite eine Flexibilisierung der Arbeit und der Arbeitszeiten, was im Falle von Telearbeit mit einer freieren Gestaltung der Arbeit und mit mehr Eigenverantwortung einhergehen kann. Die Unabhängigkeit der Telearbeitenden vergröBert sich dadurch. Auf der anderen Seite kann Flexibilisierung aber auch Anpassung und vermehrte Abhängigkeit bedeuten. Dies z. B. bei der Just-in-time-Produktion, wo die produktionssynchrone Anlieferung von Fertigungsteilen eine hochgradige Anpassung der Zulieferbetriebe an den Abnehmerbetrieb erfordert.

Im Konsum- und Freizeitbereich wird sich durch den Telematikeinsatz der Entscheidungs- und Handlungsspielraum des Einzelnen erhöhen, denn ein breiteres Informationsangebot über die Medien, die Möglichkeiten der Fernabfrage und -bestellung von Büchern oder Waren des täglichen Bedarfs sowie telekommunikative Reservationssysteme erweitern und vereinfachen die Wahlmöglichkeiten und die Suche nach Informationen. Mit dem vermehrten Einsatz von elektronischen Zahlungsmitteln (Chip- und Magnetstreifenkarten) oder von Computersystemen zur Erfassung persönlicher Daten - beispielsweise im Gesundheitswesen - wächst aber auch die Gefahr des Datenmißbrauchs. Hier gehört zur Verbesserung der Lebensqualität ein Daten- bzw. Persönlichkeitsschutz, der die informationelle Selbstbestimmung der Bürgerinnen und Bürger gewährleistet. Das heißt, daß eine Person grundsätzlich selbst über die Preisgabe und Verwendung ihrer persönlichen Daten bestimmen können sollte.

Eine zunehmende Informatisierung und Vernetzung von Unternehmen, Verwaltungen und Haushalten geht mit einer wachsenden Abhängigkeit der Gesellschaft von Computern, Netzen und Elektrizität einher. Je größer und weiter vernetzt die Computersysteme sind, desto empfindlicher trifft uns ein Fehler im System.

Wird das Bewertungskriterium Abhängigkeit aus räumlicher Sicht betrachtet, so ergibt sich eine Verstärkung der Abhängigkeit der peripheren Regionen von den Zentren. Denn Telematik fördert einen Prozeß der räumlichen Dezentralisierung bei gleichzeitiger organisatorischer Konzentration. Das heißt, sie bietet den Zentren die Möglichkeit, ihre Kontrolle über die Peripherie auszuweiten (Deutscher Städtetag, 1989, 200 ff.; ROTACH et al., 1993, 45 ff.).

Bei der Beurteilung des Beitrags der Telematik zu verbesserter Chancengleichheit stehen zwei Aspekte im Vordergrund: Die Chancengleichheit bei der Benützung von Telematikanwendungen und die Chancengleichheit verschiedener gesellschaftlicher Gruppen als Ergebnis der 
Einführung und Anwendung von Telematik. Aufgrund einer repräsentativen Bevölkerungsbefragung kommen DU MOULIN/KLAUS (1993, 53 ff.) zum Schluß, daß in der Schweizer Bevölkerung deutliche Unterschiede in Kenntnis und Nutzung neuer Telekommunikationstechniken bestehen, die zu sehr unterschiedlichen Voraussetzungen für den Umgang mit Telematik führen. Wenn in Zukunft immer mehr Dienstleistungen und Informationen über Kommunikationsmedien angeboten werden, ergeben sich für verschiedene gesellschaftliche Gruppen Nachteile, insbesondere für Frauen, ältere Menschen, Nichterwerbstätige, weniger Gebildete, sogenannte "Computeranalphabeten» und Behinderte. Chancenungleichheit und Ausgrenzungen werden dann bei Personen auftreten, die keine Möglichkeit haben, Telematik zu benützen, weil ihnen die Infrastruktur oder die finanziellen Mittel fehlen und sie dadurch wichtige Informationen nicht mehr erhalten, oder bei Menschen, die nicht fähig sind, Telematik zu benützen (Überforderung, Behinderungen, Fremdsprachige). Ein Trend zu sozialer Teilung in Bevölkerungsgruppen, die Telematik nutzen, und solche, die sie nicht nutzen, ist zu erwarten. Ein künftiges Privileg unserer Gesellschaft könnte Technikfähigkeit heißen, erworben durch Bildung und Erfahrung im Umgang mit Computern (DU MOULIN/KLAUS, 1993, 56). Telematik kann aber auch Lebenschancen verbessern, z. B. durch die Unterstützung körperlich Behinderter bei der Bewältigung ihres Alltags und durch die Möglichkeit zur Telearbeit, die je nach Behinderung einen möglichen Weg zur Reintegration in den Arbeitsprozeß darstellt (DU MOULIN, 1992).

In bezug auf die Partizipation gilt vieles, was bereits für die Unabhängigkeit genannt wurde. Mit dem Einsatz von Kommunikationsmedien kann oft ohne wesentliche Mehrkosten die Zahl der Beteiligten innerhalb eines Entscheidungsprozesses erhöht werden. So hat beispielsweise eine Erhöhung der Teilnehmerzahl an Videokonferenzen - im Gegensatz zu Geschäftsreisen - nur geringen Einfluß auf die Gesamtkosten einer Konferenz. Ob jedoch eine erhöhte Beteiligung an Videokonferenzen mehr Mitsprache oder sogar Mitbestimmung erlaubt, hängt nicht von der Technik ab, sondern von der Unternehmenskultur des jeweiligen Betriebes.

Zwischen den Betrieben werden die wirtschaftlichen Verflechtungen enger, z. B. bei der Just-in-time-Produktion, wo mehrere Zulieferbetriebe auf einen großen $\mathrm{Ab}$ nehmerbetrieb ausgerichtet sind. Hier kann im Rahmen des gesamten Produktionsprozesses die Mitbestimmung der Zulieferbetriebe derart eingeschränkt werden, daß sie ihre Konstruktionsdaten in der Form übergeben müssen, daß diese vom Abnehmerbetrieb gleich elektronisch weiterverarbeitet werden können. Damit wächst der Druck auf die Zulieferer, sich mit CAD-Systemen auszurüsten, die kompatibel sind zu jenen der Abnehmer. Im gesundheitlichen Bereich bringt ein vermehrter Telematikeinsatz einige Gefahren mit sich: Abgesehen von den bekannten Problemen bei langanhaltender Tätigkeit am Bildschirm verlagern sich generell die Belastungen bei der Arbeit von den körperlichen zu den psychischen
Belastungen. Zunehmende Automation und vermehrte Anwendung von Computern und Telekommunikation erfordern weniger Körperleistung, dafür aber mehr Aufmerksamkeit und Kontrolltätigkeit (vgl. Schweiz. Gewerkschaftsbund, 1986, 19 f.; KLAUS, 1992, 10). Mit verringerter körperlicher Tätigkeit ist weniger die Möglichkeit gegeben, Anspannungen und Streß über die Bewegung abzubauen. Für körperlich Behinderte kann dagegen eine Ablösung manueller Tätigkeiten durch Computer oder computergesteuerte Maschinen ein Vorteil sein. Die neuen Techniken vereinfachen zunächst gewisse Abläufe, ermöglichen aber zugleich neue Organisationsformen, die meist komplexer als die vorhergehenden sind und eine noch rigidere Zeitplanung erfordern. Komplexere und schnellere Produktions- und Geschäftsabläufe führen zu einer Zeitverdichtung, die dann zentral ist für die Entstehung von Streß. Psychisch unter Druck geraten wir oft auch angesichts der extremen Fehlerintoleranz mancher Computerprogramme. Ein Befehl muß absolut korrekt eingegeben werden, sonst reagiert der Computer nicht oder falsch (ROTACH et al., 1993, 70).

Zur Lebensqualität gehört auch der Aspekt der materiellen Sicherheit. Durch einen gezielten Telematikeinsatz können in vielen Betrieben Organisations- und Produktionsabläufe verbessert und Gesamtprozesse optimiert werden. Dadurch leistet die Telematik einen Beitrag zur Unternehmenssicherung und zur Steigerung der Wettbewerbsfähigkeit dieser Betriebe. Ob damit jedoch einzelne Arbeitsplätze und Einkommen gesichert sind, bleibt offen. Denn Telematik wird meist zu Rationalisierungszwecken eingesetzt. In einem ersten Schritt erfordert die Umstellung auf computergestützte Produktion, Verwaltung usw. zwar einen hohen Aufwand an Personal, Zeit und Geld für die Entwicklung der geeigneten Organisationsstrukturen sowie für die Installation der Hard- und Software und wird dadurch positive Beschäftigungswirkungen bei Hard- und Softwareherstellern und anderen Telematikproduzenten zeitigen. Die letztlich angestrebte Rationalisierung wird jedoch insbesondere im Bereich der routinisierbaren Dienstleistungen wie Auskunftsdienste, Bestellwesen und Sachbearbeitung negative Beschäftigungswirkungen auslösen (FRITSCH/EWERS, 1985, $38 \mathrm{ff}$.).

In bezug auf die Umweltverträglichkeit der Telematik interessieren wie bereits erwähnt der Ressourcenverbrauch, die Umweltbelastung und die Erhaltung des Lebensraumes. Telematik kann einerseits zu ressourcensparendem Verhalten beitragen, weil Produkte gezielter ausgewählt werden können und Informationen besser zugänglich sind. Ein Beispiel dafür sind elektronische Informations- und Reservationssysteme im Tourismus, die eine Auswahl der Feriendestination und -unterkunft per Bildschirm ermöglichen, ohne daß erst eine Flut von Prospekten durchgesehen werden muß. Andererseits können Telematikanwendungen auch zu gesteigertem Ressourcenverbrauch anregen, indem durch gezielte multimediale Vermarktung von Produkten oder ein breites Informationsangebot im Freizeit- und Tourismus- 
bereich der Konsum bzw. die Reiseaktivität angeheizt werden.

Möglichkeiten, Ressourcen einzusparen, bietet die Telematik im Dienstleistungs- und Produktionsbereich mit der besseren Koordination von Abläufen sowie Effizienzsteigerungen im Produktionsprozeß. Die direkten Energieeinsparungen durch Telematik liegen in vielen Prozessen und Anwendungen bei etwa 15\%. Ein Beispiel dafür ist der Telematikeinsatz in der Gebäudeleittechnik, der eine Energieeinsparung in Gebäuden von 15-20\% ermöglicht (ROTACH et al., 1993, 91 u. 95). Telekommunikationseinrichtungen benötigen aber bei Produktion und Betrieb selbst Energie und Rohstoffe. Nicht zu vernachlässigen ist auch der Materialverbrauch durch Nachrüsteinheiten, Ersatzteile und die äußerst kurzen Produktlebenszyklen der Endgeräte. Diese Aspekte müssen möglichen Ressourceneinsparungen durch Telematikanwendungen gegenübergestellt werden.

Als eine der bedeutendsten Möglichkeiten der Telematik zur Reduktion des Ressourcenverbrauchs und der Emissionen wird häufig die Substitution von Verkehr durch Telekommunikation genannt, etwa der Ersatz von Faceto-face-Kontakten durch Telefon- oder Videokonferenzen. Vor allem im geschäftlichen Bereich mit seinen hohen Anteilen an zeitkostensensiblen Kommunikations- und Reiseaktivitäten ist ein gewisses Substitutionspotential der Telematik zu vermuten. Diverse Studien (SCHULTE, 1993; HENCKEL, 1990; FRITSCH/ EWERS, 1985) weisen jedoch darauf hin, daß zum einen nicht jede Art von Informationsaustausch durch Telematik ersetzbar ist und zum andern die Nutzung der Telematik neue Kommunikationsbedürfnisse (z. B. Reisen) auslösen kann.

Um eine Reduktion der Umweltbelastung zu erreichen, kann Telematik gezielt für die Realisierung möglichst geschlossener Kreisläufe eingesetzt werden. Der Telematik kommen Überwachungs- und Steuerungsfunktionen zu, indem beim Überschreiten bestimmter Grenzwerte oder Qualitätsmerkmale automatisch Maßnahmen eingeleitet werden. Die Grenzwerte und die Art der Reaktionen (z. B. Fahrverbote, Abschalten der Produktionsanlage usw.) müssen jedoch vorgängig festgelegt werden.

Zur Erhaltung des Lebensraumes sind zwei Aspekte zentral: der haushälterische Umgang mit dem Boden in Siedlungsgebieten sowie der Nutzungsverzicht oder die Rücknahme der Intensität der Flächennutzung in unbebauten Gebieten, also in Forst-, Landwirtschafts- und Schutzgebieten. Bei der Verringerung der Nutzungsintensität in Land- und Forstwirtschaft bleibt der Beitrag der Telematik beschränkt auf schnellere und umfassendere Informationsangebote und gezielteren, verringerten Dünger- oder Pestizideinsatz. Telematikanwendungen können hier nur Hilfsmittel sein, um einen ersten Schritt in Richtung Extensivierung zu tun.

Dagegen können Telematikanwendungen im Hochwasserschutz oder in der Lawinenfrühwarnung den Schutz des Kulturlandes verbessern helfen. Die Fernablesung, Verarbeitung und Weiterleitung von meteorologischen und hydrologischen Daten allein kann aber Über- schwemmungen nicht abwenden. Erst im Zusammenwirken mit baulichen Maßnahmen wie Abflußvergrößerungen und Wehren oder organisatorischen Vorkehrungen (Evakuationen) können Hochwasser- oder andere Naturereignisse verhindert oder deren Auswirkungen begrenzt werden.

Zum haushälterischen Umgang mit dem Boden ist anzufügen, daß auf der einen Seite durch den Einsatz von Telematik gerade in Industriebetrieben Flächeneinsparungen respektive eine erhöhte Nutzung und Wertschöpfung bei gleichem Flächenverbrauch möglich sind. Auf der anderen Seite stellt die neue Technologie neue Anforderungen an den Fabrikbau bezüglich dessen Multifunktionalität und der Ver- und Entsorgungseinrichtungen unterschiedlichster Art (Datenleitungen, Kühlung, Einrichtungen zur automatischen Verkettung der Produktionsschritte usw.). Diese neuen Anforderungen erschweren in vielen Fällen die Umnutzung alter Gebäude und machen einen Neubau erforderlich. Daher ist eine weitere Zunahme des Flächenverbrauchs zu erwarten (Technikentwicklung und Raumstruktur, 1987, 178 ff.).

Weitere Hinweise auf einen zunehmenden Flächenbedarf der Haushalte und Betriebe sind unter anderem bei HENCKEL/NOPPER/RAUCH, 1984, und FRITSCH/EWERS, 1985, zu finden. Sie argumentieren mit dem Bedeutungsverlust der Kernstädte gegenüber ihrem Umland durch eine verbreitete Anwendung der Telematik insbesondere im Dienstleistungsbereich - Teleshopping, Telebanking und andere mediale Dienstleistungen - sowie durch Verbreitung der Telearbeit und erwarten kleinräumlich eine Ausdehnung der Einzugsgebiete der Städte, also eine fortschreitende Suburbanisierung. In großräumlicher Hinsicht sehen sie eine Intensivierung der interregionalen Arbeitsteilung, dabei hauptsächlich eine Konzentration von wichtigen Entscheidungs- und Kontrollfunktionen auf Verdichtungsgebiete mit internationaler Bedeutung, und befürchten Entleerungstendenzen für den ländlichen Raum. Der Einsatz der Telematik wird einen Prozeß organisatorischer Konzentration bei gleichzeitiger räumlicher Dezentralisierung fördern.

Die obigen Aussagen zur Sozial- und Umweltverträglichkeit der Telematik zeigen die ambivalenten Wirkungen dieser Technologie auf. Sie ergeben kein eindeutiges Bild, denn die Telematik ist nicht per se sozial- oder umweltverträglich. Bei der Frage nach der Verträglichkeit für Mensch, Gesellschaft und Umwelt spielt eine Vielzahl von Entscheiden mit. Es ist nicht nur eine Frage des Was (für eine Technik wird angewandt), sondern auch des Wie (wird die Technik angewandt) und des Wozu (wird die Technik angewandt). Entscheidend für die Auswirkungen der Telematik ist demnach, mit welchen Zielsetzungen und unter welchen wirtschaftlichen, sozial- und umweltpolitischen Rahmenbedingungen sie eingesetzt wird.

Die TA-Forschung kann lediglich Chancen und Risiken einer Technologie aufzeigen und ihre Wirkungen in möglichst vielen Bereichen beurteilen. Damit liefert sie Entscheidungsgrundlagen für eine Technologiepolitik, die sich fragen muß, welche Zukunft sie ansteuern will. Zen- 
tral ist dabei die Frage, ob sich eine bestimmte Technologie im Hinblick auf die gewünschte Entwicklung steuern läßt und wie dies allenfalls möglich wäre. TechnikfolgenAbschätzung soll der Politik nicht die Entscheidungen abnehmen, sondern die Entscheidungen hinsichtlich der Ausrichtung der Technologiepolitik erleichtern. Der Beitrag, den Geographinnen und Geographen dabei leisten können, umfaßt vor allem die Erarbeitung räumlicher, aber auch sozialer und ökologischer Bewertungskriterien im Hinblick auf eine gewünschte Entwicklung sowie die Synthese und Bewertung räumlicher Wirkungen von Technologien unter Einbezug bestehender regional-, umwelt- und wirtschaftspolitischer Ziele und Rahmenbedingungen.

\section{Anmerkungen}

' Die Begriffe "Technik" und "Technologie" werden heute oft synonym verwendet, wie die Definitionen im Brockhaus-Lexikon zeigen: "Technik, konstruktives Schaffen von Erzeugnissen, Vorrichtungen und Verfahren unter Benutzung der Stoffe und Kräfte der Natur und unter Berücksichtigung der Naturgesetze ... Die Entwicklung der T. zielt darauf ab, die Erzeugung von Stoffen und Gütern ohne Beeinträchtigung der Humanität am Arbeitsplatz und mit möglichst großer Schonung der Umwelt und der Ressourcen immer zweckentsprechender und wirtschaftlicher zu gestalten. Besonders in diesem Zusammenhang wird heute auch der Begriff Technologie verwendet." - "Technologie, im 18. und 19. Jh. die Lehre von der Entwicklung der Technik in ihren gesellschaftlichen Zusammenhängen; in den Ingenieurwissenschaften in DtI. eingeschränkt auf die Bedeutung Verfahrenskunde. Die heutige Wiederaufnahme des älteren, weiteren Bedeutungsinhalts deutet auf ein wachsendes Bewußtsein der engen Verflechtung der Technik mit anderen gesellschaftlichen Faktoren hin. T. wird heute auch häufig im Sinne von Technik verwendet.) (Zitat: Brockhaus, Naturwissenschaften und Technik, Bd. 5, Wiesbaden 1983, S. 105 u. 107)

${ }^{2}$ Der Begriff Telematik entstand aus der Verschmelzung der Bereiche Telekommunikation und Informatik. Er wird in der Literatur oft synonym verwendet mit Bezeichnungen wie "Informations- und Kommunikationstechnologien", "moderne Telekommunikationstechniken" oder "neue Informationstechnologien".

\section{Literatur}

BALLERSTEDT, E., et al. (1982): Forschungsbericht DV 82002 Datenverarbeitung - Studie über Auswahl, Eignung und Auswirkungen von informationstechnisch ausgestalteten Heimarbeitsplätzen (Bundesministerium für Forschung und Technologie), Tübingen.

BERGER, R. (1991): Politik und Technik. Der Beitrag der Gesellschaftstheorien zur Technikbewertung, Opladen.
BÖHRET, C., FRANZ, P. (1982): Technologiefolgenabschätzung. Institutionelle und verfahrensmäßige Lösungsansätze, Frankfurt a. M., New York.

BÖTTGER, B., FIEGUTH, G. (Hg.) (1992): Zukunft der Informationstechnologie / für die Grünen / Bündnis 90, Münster.

BUCHS, T.(1992): Technology Assessment: Expériences occidentales et défis actuels. Forschungspolitische Früherkennung, Nr.131, hg. v. Schweizerischen Wissenschaftsrat, Bern.

Deutscher Städtetag (Hg.) (1989): Telematik und Stadtentwicklung, DST-Beiträge zur Stadtentwicklung und zum Umweltschutz, Reihe E, Heft 17, Köln.

DGB-Bundesvorstand, Abteilung Technologie/HdA (1991): Für eine soziale Gestaltung der Telekommunikation - Thesen und Vorschläge des DGB, Düsseldorf.

DU MOULIN, S. (1992): Telearbeitsplätze für Behinderte, Untersuchungsbericht zum Einzelprojekt SUR 14, Wissenschaftliche Begleituntersuchung zum Projekt "Kommunikations-Modellgemeinden der Schweiz (KMG)", Zürich.

DU MOULIN, S., KLAUS, P. (1993): Kenntnis und Nutzung der Telekommunikation in der Schweizer Bevölkerung, Auswertung der WBU-Bevölkerungsumfragen unter dem Aspekt der Chancengleichheit. Wissenschaftliche Begleituntersuchung zum Projekt “Kommunikations-Modellgemeinden der Schweiz (KMG)", Zürich.

FRITSCH, M., EWERS, H.-J. (1985): Telematik und Raumentwicklung. Mögliche Auswirkungen neuer Telekommunikationstechniken auf die Raumstruktur und Schlußfolgerungen für die raumbezogene Politik, Bonn.

GARBE, D., LANGE, K. (Hg.) (1991): Technikfolgenabschätzung in der Telekommunikation. Schr.-R. des Wissenschaftlichen Instituts für Kommunikationsdienste, Bd. 12, Berlin, Heidelberg, New York.

HENCKEL, D.(Hg.)(1990): Telematikund Umwelt. Deutsches Institut für Urbanistik (difu), Berlin.

HENCKEL, D., NOPPER, E., RAUCH, N. (1984): Informationstechnologie und Stadtentwicklung. Schriften des Deutschen Instituts für Urbanistik, Bd. 71, Stuttgart.

HEPPNER, A. (1989): Abschätzung und Bewertung von Technikfolgen - zur Diskussion über die Informations- und Kommunikationstechnologien in der Bundesrepublik Deutschland, Regensburg.

KLAUS, P. (1992): Serveur Technique du Bâtiment. Schlußbericht zum Einzelprojekt SIE 24. Wissenschaftliche Begleituntersuchung zum Projekt "Kommunikations-Modellgemeinden der Schweiz (KMG)", Zürich.

KORNWACHS, K. (Hg.) (1991): Reichweite und Potential der Technikfolgenabschätzung, Stuttgart.

KORTE,W. B., ROBINSON, S., STEINLE, W. J. (Hg.) (1988): Telework - present situation and future development of a new form of work organization, Amsterdam, New York, Oxford, Tokio.

KUHLMANN, A. (1977): Alptraum Technik? Zur Bewertung der Technik unter humanitären und ökonomischen $\mathrm{Ge}$ sichtspunkten, Darmstadt, Berlin.

LENK, H., ROPOHL, G. (Hg.) (1987): Technik und Ethik, Stuttgart.

LENK, T. (1989): Telearbeit - Möglichkeiten und Grenzen einer telekommunikativen Dezentralisierung von betrieblichen Arbeitsplätzen, Berlin.

METTLER-MEIBOM, B. (1986): Breitbandtechnologie: über die Chancen sozialer Vernunft in technologiepolitischen Entscheidungsprozessen (Beiträge zur sozialwissenschaftlichen Forschung, Band 79), Opladen. 
PASCHEN, H., GRESSER, K., CONRAD, F. (1978): Technology Assessment - Technologiefolgenabschätzung, Frankfurt a. M., New York.

PETERMANN, T. (Hg.) (1991): Technikfolgen-Abschätzung als Technikforschung und Politikberatung, Frankfurt a. M./ New York.

RAPP, F., MAI, M. (Hg.) (1989): Institutionen der Technikbewertung. Standpunkte aus Wissenschaft, Politik und Wirtschaft. Vorträge und Diskussionen, VDI-Reihe Der Ingenieur in Beruf und Gesellschaft, Düsseldorf.

ROPOHL, G., et al. (1978): Maßstäbe der Technikbewertung. Vorträge und Diskussionen, hg. v. Verein Deutscher Ingenieure, Düsseldorf.

ROTACH, M., et al. (1993): Telematik und qualitatives Wachstum, Wissenschaftliche Begleituntersuchung zum Projekt "Kommunikations-Modellgemeinden der Schweiz (KMG)", Synthesebericht, Zürich.

SCHULTE, R. (1993): Substitut oder Komplement - die Wirkungsbeziehungen zwischen der Telekommunikationstechnik Videokonferenz und dem Luftverkehrsaufkommen deutscher Unternehmen, Bonner Geographische Abhandlungen, H. 88, Bonn.

Schweizerische Bundeskanzlei (Hg.) (1988): Bericht über die Legislaturplanung 1987-1991, Bericht des Bundesrates an die Bundesversammlung vom 18.1.1988, Bern.

Schweizerische Bundeskanzlei (Hg.) (1992): Bericht über die Legislaturplanung 1991-1995, Bericht des Bundesrates an die Bundesversammlung vom 25.3.1992, Bern.
Schweizerischer Gewerkschaftsbund (Hg.) (1986): Arbeitszeit und Gesundheit, Stellungnahme des Schweizerischen Gewerkschaftsbundes, Bern.

SCHWOHNKE, A., WICKE, H.-G. (1986): Teleheimarbeit als neue Rationalisierungsstrategie - Analyse und Perspektiven einer neuen Form der Arbeitsorganisation, Köln.

SWR Schweizerischer Wissenschaftsrat (Hg.) (1992): Programm TA, Technology Assessment $(=1$. Ausschreibung zum Programm TA), TA 1b/1992.

Technikentwicklung und Raumstruktur (1987): Perspektiven für die Entwicklung der wirtschaftlichen und räumlichen Struktur der Bundesrepublik Deutschland, Referate und Diskussionsberichte anläßlich der Wissenschaftlichen Plenarsitzung 1986 in Nürnberg, Veröffentlichungen der Akademie für Raumforschung und Landesplanung, Hannover.

VDI-Technologiezentrum Physikalische Technologien ( $\mathrm{Hg}$.) (1992): International vergleichende Analyse der Institutionalisierung von Technikfolgenabschätzung - zwischen Etablierung, auf Rollensuche und ungewissen Chancen, Düsseldorf.

Verein Deutscher Ingenieure, Ausschuß "Grundlagen der Technikbewertung": Vorentwurf für eine Richtlinie "Empfehlungen zur Technikbewertung" 1986, in: LENK, H., ROPOHL, G. (Hg.) (1987): Technik und Ethik, Stuttgart, 297-325.

WITT-BARTHEL, A. (1992): Chancen sozialorientierter Technikgestaltung, Politische Ansätze und Gestaltbarkeit der Informationstechnik in der sozialen Sicherung, Opladen. 